\title{
The Influence of Balance Hole Position of Water Pump on Axial Force and External Characteristics
}

\author{
Zhang Jie, Zhao Bo \\ Automobile Engineering College, Shanghai University of Engineering Science, Shanghai, China
}

Email address:

zhangjie12012@163.com (Zhang Jie)

\section{To cite this article:}

Zhang Jie, Zhao Bo. The Influence of Balance Hole Position of Water Pump on Axial Force and External Characteristics. International Journal of Science, Technology and Society. Vol. 4, No. 2, 2016, pp. 21-24. doi: 10.11648/j.ijsts.20160402.11

Received: February 24, 2016; Accepted: March 5, 2016; Published: March 17, 2016

\begin{abstract}
The method of balancing the axial force of water pump by balance hole is taken as a research object. Based on the combined CFD technology and experimental research, the relationship between the radial position of balance hole and the effect of axial force, external characteristics of the pump is studied in-depth. Research shows that, the effect of balance hole location on the axial force and the external characteristics have certain law, which effect is significant in balancing the interior pressure; with the increase of radial radius of the balance hole location, the overall balance of interior pressure is increased, the greater the axial force is; the smaller radial radius is, the better the effect of axial force balance is, but leakage may increase and the stroke and efficiency is decreased slightly.
\end{abstract}

Keywords: Water Pump, Axial Force, Balance Hole Position, Numerical Simulation, External Characteristics

\section{Introduction}

Water pump impeller wear-ring clearance produced not only volumetric leakage, but also changed the internal flow of water pump, so as to have important influence on water pump external characteristic. Because of the wear-ring clearance size smaller, complex flow, difficult to study, so, based on the experimental method, the influence of the wear-ring clearance on the external characteristics of the water pump was studied, and the numerical simulation based on the CFD usually ignores the existence of the wear-ring clearance. Therefore, it is very meaningful that the test method and numerical simulation method are used to study the external characteristics with wear-ring clearance of water pump.

H. F. Black, through theoretical analysis and experimental study on the fluid force in the annular clearance seals of the water pump, points out that the important influence on the rotor vibration of water pump [1]. Toshio Hirano et al. Numerical simulation of the labyrinth seal was used to study the effect of leakage flow and clearance flow on the rotor stress [2]. Chen Yu et al studied the effect of the test method on the characteristics of the water oil pump when it was working with pure water, the difference between the front and rear ring on the characteristics of the water pump was compared [3]. In addition, other scholars study the wear-ring clearance on water pump leakage loss and mechanical efficiency influence and so on content $[3 \sim 6]$.

Comprehensive on the wear-ring clearance of the research work above, we can see that its main focus on the influence of collar clearance on water pump volume loss and mechanical loss, it ignored the influence of hydraulic loss, which had few study about how to determine the actual operation of the water pump in the process of wear-ring clearance between the radius value. This paper studied the wear-ring clearance has impact on the hydraulic loss in water pumps; a comparison between test value and the calculated value shows how to determine optimal opening ring scheme, in the end, the author summarized the characteristics of water pump.

\section{Calculation Model and Grid}

Research object for IS80-50-220 water pump, the leaf number is 6 , the specific speed of 59; design parameters for flow $54 \mathrm{~m} 3 / \mathrm{h}$, head $61 \mathrm{~m}$, speed $2900 \mathrm{rpm}$. in order to investigate the influence of different clearance variation on the performance of the pump, the calculation model using the three schemes: changing front and rear the wear-ring clearance dimension; changing front the wear-ring clearance dimension, rear wear-ring clearance dimension remains 
constant; changing rear the wear-ring clearance dimension, front wear-ring clearance dimension remain invariant. Letter $b$ indicates that the dimension of the front and rear rings were changed simultaneously. Letter h denotes the change only rear the wear-ring clearance radial dimension. The original design of the pump wear-ring clearance was $0.15 \mathrm{~mm}$, reserved pump mouth ring does not move to reduce the upper opening of the impeller ring diameter. the wear-ring clearance radial dimension changed value for $0.25,0.35,0.5$. In order to compare and ignore the wear-ring clearance model, there were calculated using the $\mathrm{b}_{0}$ representation. [8]

Computation model and grid was shown in figure 1 (a), the number of grid total of 120 140 million; Figure 1 (b) for the wear-ring clearance grid.

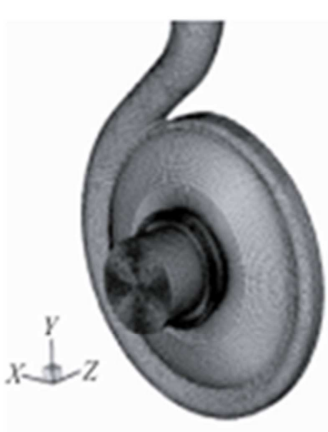

(a)

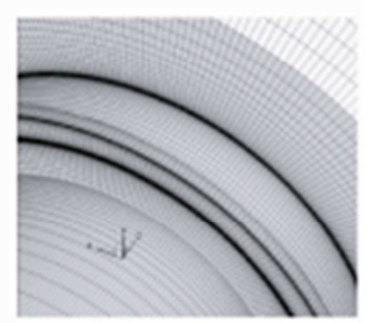

(b)
Fig. 1. Computational model and mesh.

\section{Numerical Simulation Settings}

Simulation calculation select the separation of the solution, it assumes that the water pump flow within the constant. The three-dimensional turbulent flow in an engine cooling water pump with the impeller balance hole and clearance of wear-rings, which under the external characteristics of the influence, was simulated employing the time-averaged N-S equations, the standard $\mathrm{k}-\varepsilon$ turbulence model by computational fluid dynamics software FLUENT. In the difference scheme, the pressure terms were used in the standard format, the velocity term, the turbulent kinetic energy term and the turbulent viscosity coefficient are all in the first order upwind difference scheme. The coupling model between the rotating region of the impeller and the static region of the spiral case and the water suction chamber were selected the multiple reference frame model [7-8]. Inlet boundary condition by setting velocity-inlet; the exit boundary condition by setting outflow; each interface was set to Interface. Impeller flow channel region using the rotation of the coordinate system (MRF), according to the law of the right hand along the $\mathrm{Y}$ axis in the direction of rotation [9], the speed is $2900 \mathrm{r} / \mathrm{min}$. Simulation of the flow field at different working conditions by changing the inlet velocity.

\section{Results and Analysis}

The numerical simulation of 6 working conditions were carried out according to different clearance. Based on the calculation results, the external characteristic curve and the internal flow field structure of the pump were obtained. Table 1 compares the calculation results with the test results for 6 working conditions, and the numerical results were not corrected efficiency. As can be seen from table 1, there is no gap between the calculated results larger than which have the wear-ring clearance; the calculated results compared with the experimental results under the maximum error was not more than $5 \%$, which in the acceptable range.

Table 1. Comparison between computation and experiment.

\begin{tabular}{|c|c|c|c|c|c|c|c|c|c|}
\hline \multirow{2}{*}{$\mathbf{Q} / \mathbf{Q}_{\mathbf{0}}$} & \multicolumn{4}{|l|}{$\mathbf{H} / \mathbf{m}$} & \multicolumn{3}{|l|}{$\mathbf{P} / \mathbf{k w}$} & \multicolumn{2}{|l|}{$\eta / \%$} \\
\hline & $\operatorname{Exp}$ & b 0.15 & bo & Exp & b0.15 & bo & Exp & b0.15 & bo \\
\hline 0.6 & 64.2 & 65.89 & 65.71 & 10.66 & 10.36 & 9.24 & 52.0 & 54.37 & 61.40 \\
\hline 0.8 & 63.0 & 65.67 & 65.80 & 11.52 & 11.82 & 10.49 & 63.0 & 63.98 & 72.71 \\
\hline 1.0 & 61.0 & 63.97 & 65.32 & 13.30 & 13.87 & 12.47 & 66.0 & 66.39 & 75.38 \\
\hline 1.1 & 60.0 & 63.02 & 65.12 & 13.77 & 14.45 & 13.49 & 69.0 & 67.81 & 76.43 \\
\hline 1.2 & 59.0 & 61.94 & 64.26 & 14.66 & 15.38 & 14.43 & 69.5 & 68.49 & 76.91 \\
\hline 1.3 & 58.0 & 60.41 & 62.83 & 15.51 & 16.28 & 15.32 & 70.0 & 68.72 & 76.76 \\
\hline
\end{tabular}

Figure 2, 3respectively under different clearance changes in the pump head, power, efficiency characteristic curve. From Figure 2, we can see that with the increase of the gap, pump head and efficiency are decreased, power are on the rise, which the same operating point head the biggest change value to $1.54 \mathrm{~m}$, the biggest change in the power value to $0.67 \mathrm{kw}$, the biggest change in efficiency value reached $5.2 \%$. Due to the increase of the radial force, the eccentric vortex of the rotor will be increased, which will lead to the increase of the mechanical loss of the rotor system. This shows that with decrease of the wear-ring clearance value, reduced the radial force and the eccentricity of the rotor vortex move weaken and reduced the friction between the rotor and the pump body, which eventually leading to reduce the mechanical losses of the whole pump while mechanical efficiency increases, which in reference [10] by methods of test results are consistent. Under the design condition, the predicted head $\mathrm{H}$ curve with the change of the position of the balance hole is shown in figure 3. The head curve shown in figure H-R0 was similar to that of the axial force curve F-R0 in Figure 2, which shows that the lift of the water pump was higher with the increase of the radial radius of the balance hole position. 


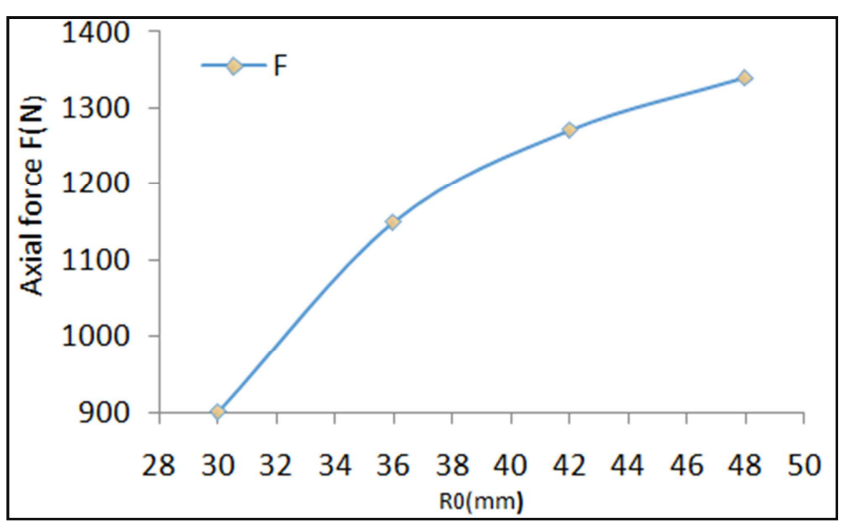

Fig. 2. The variation curve of the axial force $F$ with the position of the balance hole under the design conditions.

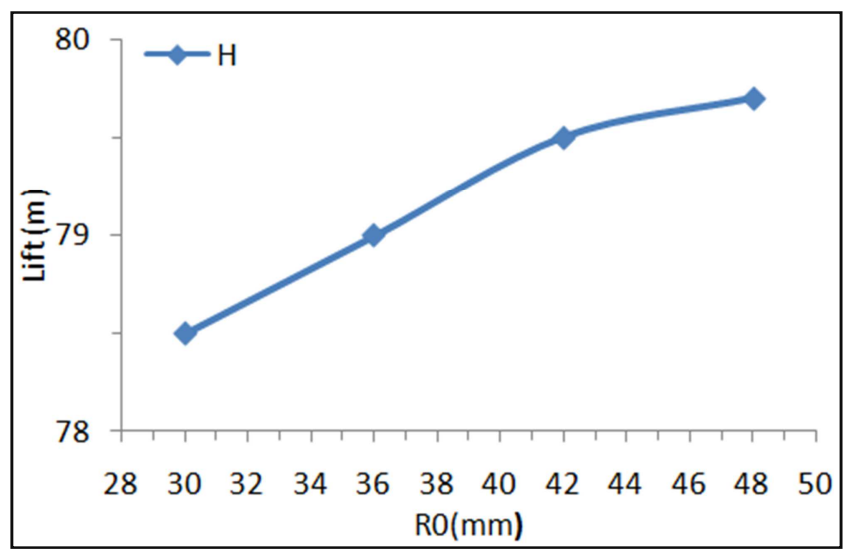

Fig. 3. The variation curve of head $H$ with balance hole position in design condition.

Due to the most hours of the balance hole position of radial radius, indoor pressure through a smaller axial force was generated, but this time after the sealing rings on both sides of the fluid pressure, so the leakage rate was larger; in addition, leakage flow of impeller suction inlet fluid flow state of damage was more serious, thus, pump head, minimum efficiency lowest. When the balance hole position of radial radius increases, the balancing chamber pressure also increases, rear sealing rings on both sides of the pressure difference decreases, balance hole leakage was reduced; leakage flow of impeller suction inlet of fluid flow was less affected and pump head was larger and the efficiency higher. Similarly, in other conditions, the balance chamber pressure, axial force, head and other changes have similar laws. Therefore, the balance hole position selection has an important influence on the axial force and the overall performance of the water pump.

\section{Experimental Research}

In order to understand the influence of the position of the balance hole on the axial force and the external characteristic, the axial force test was carried out on the prototype model. As shown in figure 4. According to the research project, the prototype of four different balance hole positions were made, and the test results were compared with the simulation results.
The axial force was compared showing in Figure5. The result of the axial force and external characteristics of the water pump with the change of the position of the balance hole, which was consistent with the result of numerical simulation, verified the accuracy of the numerical simulation. The experiment shows that the axial force was slightly larger than the computed value, because it was in the ideal state in the simulation process. In addition, the test head not only due to a small deviation, but also by other devices, so it was difficult to measure effective value. Reasonable proposals were put forward to reduce the hydraulic losses of automotive water pump parts. The research results can guide the development and design of the pump as well as the congener products.

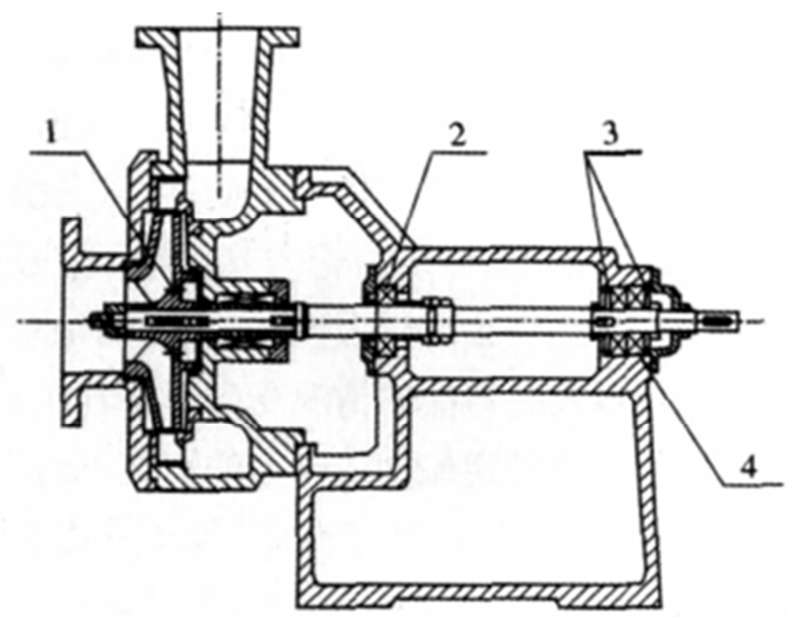

1. balance hole 2. radial bearing 3. axial proving ring 4.thrust bearing

Fig. 4. Structure of axial force testing device.

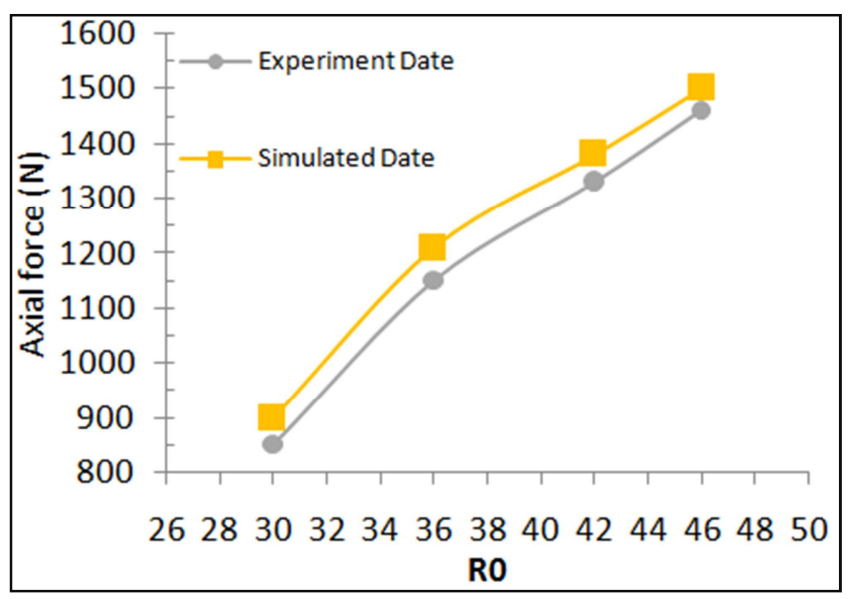

Fig. 5. Comparison diagram of axial force under design conditions.

\section{Concluding Remarks}

(1) The front and rear wear-ring clearance also change the pump characteristic curve, leakage volume has the most significant effect, only the front wear-ring clearance change times, the rear wear-ring clearance change minimum, the analysis shows that front wear-ring clearance variations play main role;

(2) The greater the radial radius of the balance hole, the 
greater the overall pressure in the chamber and the distribution law of the pressure from the hub to the mouth ring gradually increases;

(3) With the decrease of the radial radius of the position of the balance hole, the axial force becomes smaller, and the effect of the axial force of the balance hole was better. The balance hole position was close to the hub and the axial force difference was larger than that of the wheel hub;

(4) The change of the balanced aperture to the position has great influence on the axial force and the external characteristic, there was a certain regularity.

\section{References}

[1] Black H F. Effects of hydraulic forces in annular pressure $H$ Black F. Effects of hydraulic forces in annular pressure seals on the vibrations of water pump rotors On the vibrations of water pump rotors seals [J]. Journal of Mechanical Engineering Science, 1969, 11(2): 206-213. Journal of Mechanical Engineering Science J, 1969, 11 (2): 206-213.

[2] Toshio Hirano, GUO Zenglin, R. Gordonkirk. Hirano Toshio, Zenglin GUO, Gordonkirk. Application of Computational Fluid Dynamics Analysis for Computational. Fluid Dynamics Analysis for of Rotating Machinery - Part II: Labyrinth Seal Analysis Machinery Part II: Labyrinth Seal Rotating Analysis $[\mathrm{J}]$. Transactions of the ASME, 2005, 127: 820-826. Transactions of the ASME J, 2005, 820-826. 127.

[3] Chen Yu, Fei Zhentao, Cai Yongxiong, et al. Effect of inlet ring clearance on the performance of water oil pump [J]. Fluid machinery, 2006, 34 (1): 1-5.

[4] Tam L T, Przekwas A J, Muszynska A, et al. Numerical and Analytical Study of Fluid Dynamic Forces in Seals and Bearings [J]. ASME J. Vibr. Acoust, 1988, 110: 315-325.

[5] Childs D W. water acceleration modes for incompressible fluid in the leakage annulus between a shrouded pump impeller and its housing [J]. Journal of Vibration, Acoustics, Stress and Reliability in Design, 1991, 113(2): 209-218.

[6] Wang Yang, Zhang Xiang. The efficiency of water pump with low specific speed in impeller clearance of impeller. The influence of J. Drainage and irrigation machinery, 2008, 26 (6): 27-30.

[7] Wu Da Zhuan, Xu Binjie, Wu Peng, et al. Internal clearance flow of multi-stage water pump. Journal of Zhejiang University (Engineering Science Edition), Journal of (Engineering Science), 2011, 45 (8): 1393-1398.

[8] M. Aighir, F. Billy, G. Pineau, J. Frene and A. Texier, Theoretical analysis of textured "damper" annular seals, Journal of Tribology, 129 (3) (2007) 669-678.

[9] T-W. Ha and B. S. Choe, Prediction of rotor dynamic coefficients for high-performance-pump seal using CFD analysis, Journal of the KSTLE, 26 (1) (2010) 37-43.

[10] B. S. Choe and T-W. Ha, Prediction of leakage and rotor dynamic coefficients for annular-type-plain-pump seal using CFD analysis, IFToMM-Conference on Rotor dynamics (2010) 470-474. 